\title{
Marcadores de estrés oxidativo en hipertensión leve
}

\author{
Raquel Oré ${ }^{1}$, Rubén Valdivieso ${ }^{1}$, Silvia Suárez ${ }^{1}$, Doris Huerta ${ }^{1}$, Marco Núñez $^{1}$, \\ Jorge Durand ${ }^{2}$
}

Resumen

Introducción: La hipertensión es un factor de riesgo cardiovascular y en 90\% de los casos se desconoce el mecanismo que la inicia. Recientemente, se considera que la hipertensión es un síndrome de anormalidades metabólicas y estructurales, en el que las especies reactivas derivadas del oxígeno (EROs) juegan un papel fisiopatológico preponderante en su desarrollo. Objetivo: Determinar los niveles séricos de zinc, selenio y magnesio en personas normotensas e hipertensas. Asimismo, valorar la actividad de la enzima superóxido dismutasa (SOD) y los niveles de sustancias reactivas al ácido tiobarbitúrico (TBARS) en ambos grupos de estudio. Diseño: Estudio analítico observacional, tipo casos y controles. Lugar: Centro de Investigación de Bioquímica y Nutrición, Universidad Nacional Mayor de San Marcos. Material biológico: Sangre de pacientes normotensos y con hipertensión leve. Intervenciones: Se obtuvo las muestras de sangre de 20 pacientes normotensos y 20 con hipertensión leve, con edades entre 50 y 60 años, después de un ayuno de 12 horas, realizándose el estudio en suero y sangre total. Principales medidas de resultados: Variación de niveles de TBARS, oligoelementos (Se, Zn y Mg), medición de actividad de SOD eritrocitario. Resultados: En el grupo de hipertensos, se obtuvo incremento significativo de magnesio y TBARS y disminución significativa de zinc y de la actividad de SOD. Conclusiones: Se demuestra el compromiso del estrés oxidativo en pacientes con hipertensión leve. La hipermagnesemia podría explicarse por el daño a los elementos formes de la sangre.

Palabras clave Estrés oxidativo; hipertensión; superóxido dismutasa; selenio; magnesio; zinc, peroxidación de lípido.

\section{Oxidative stress markers in mild hypertension}

Abstract

Introduction: Hypertension is a cardiovascular risk factor and the initial mechanism is unknown in $90 \%$ of the cases. Currently hypertension is considered a syndrome with metabolic and structural abnormalities where oxygen reactive species (ORS) play a preponderant pathophysiological role. Objective: To determine zinc, selenium and magnesium serum levels in hypertensive and normotensive subjects, and to measure superoxide dismutase (SOD) activity and thiobarbituric acid reactive substances (TBARS) levels in both groups. Design: Analytical observational, case-control study. Setting: Biochemistry and Nutrition Research Center, Universidad Nacional Mayor de San Marcos, Lima, Peru. Biological material: Normotensive and mild hypertensive patients blood samples. Interventions: Blood samples

1 Centro de Investigación de Bioquímica y Nutrición. Facultad de Medicina, Universidad Nacional Mayor de San Marcos. Lima, Perú.

2 New York University. New York, USA. from 20 normotensive subjects and 20 patients with mild hypertension aged 50 to 60 years were obtained after 12-hour fasting. We studied serum and whole blood. Main outcome measures: Serum determination of TBARS and oligoelements ( $\mathrm{Zn}$ and $\mathrm{Mg}$ ), measurement of SOD erythrocytic activity. Results: In the hypertensive group we obtained significant increase in magnesium and TBARS, and significant decrease in zinc and SOD activity. Conclusions: We demonstrated the occurrence of oxidative stress in mild hypertensive patients. Hypermagnesemia could be explained by blood elements injury.

Key words: Oxidative stress; hypertension; superoxide dismutase; selenium; magnesium; zinc; lipid peroxidation.

\section{INTRODUCCIÓN}

La hipertensión es un factor de riesgo cardiovascular muy importante y en el $90 \%$ de los casos se desconoce el mecanismo que la inicia. La denominada hipertensión arterial esencial (HTA 
esencial) constituye la enfermedad crónica más frecuente en la sociedad moderna y es también uno de los factores de riesgo cardiovascular primario ${ }^{(1)}$. Recientemente, se ha considerado que la HTA esencial es un síndrome de anormalidades metabólicas y estructurales (genéticas y adquiridas); dentro de las alteraciones metabólicas han surgido evidencias que indican que las especies reactivas derivadas del oxígeno (EROs) juegan un papel fisiopatológico preponderante en el desarrollo de la hipertensión $\left(^{2}\right)$.

Esto se debe, en gran medida, al exceso de $\mathrm{O}_{2}{ }^{-}$ y la disminución de la liberación de NO (óxido nítrico) en la remodelación cardiovascular y en la vasculatura del riñón, mediado por los EROs $\left({ }^{3}\right)$. En la hipertensión humana, los marcadores biológicos del estrés oxidativo sistémico están elevados $\left.{ }^{4}\right)$. Así, el tratamiento con superóxido dismutasa (SOD) y otros antioxidantes análogos mejoran las funciones vasculares y renales $\left({ }^{5,6}\right)$.

El estrés oxidativo se refiere a un desequilibrio entre la generación de EROs sobre las defensas naturales, enzimáticas y no enzimáticas, produciendo alteraciones transitorias o definitivas de la relación estructura-función en los niveles de organización celular. Estudios clínicos han mostrado que en humanos con HTA esencial ocurre un incremento de la producción de EROs y una reducción de las defensas antioxidantes $\left({ }^{4,5}\right)$.

Investigaciones recientes en pacientes con HTA esencial evidencian una situación de estrés oxidativo, con incremento de la concentración sanguínea de sustancias reactivas al ácido tiobarbitúrico (TBARS), como indicador de peroxidación lipídica y reducción de las actividades antioxidantes de las enzimas superóxido dismutasa (SOD), glutatión peroxidasa $(\mathrm{Gpx})$ y catalasa, en sangre total $\left({ }^{6-9}\right)$. Así mismo, estudios realizados por Ward y colaboradores $\left({ }^{10}\right)$ encontraron una disminución de barredores de EROs, como la vitamina E y el glutatión reducido, que puede contribuir al daño oxidativo que se observa en la hipertensión en humanos.

El magnesio afecta la presión arterial, modulando el tono muscular y la reactividad. Actúa como un antagonista del canal de calcio, estimula la producción de vasodilatadores -como las prostaciclinas y el óxido nítrico- y altera las respuestas vasculares a los agonistas vasoactivos. La deficiencia de magnesio ha sido implicada en la patogénesis de hipertensión con estudios epidemiológicos y experimentales, demostrando una correlación inversa entre presión arterial y niveles de magnesio sérico $\left({ }^{11}\right)$.

Las actividades de las enzimas de defensa antioxidante -como la SOD citosólica, que es la isoenzima dependiente de zinc y cobre, y la Gpx, dependiente de selenio-, se encuentran disminuidas en los pacientes con HTA esencial $\left(^{8}\right)$. En el presente estudio se investigó los niveles de elementos como el zinc y selenio plasmáticos, como indicadores indirectos de las actividades de ambas enzimas SOD y Gpx. Igualmente, se midió la actividad de la SOD y se evaluó los niveles de TBARS, como indicador de peroxidación lipídica.

Debido a que diversos estudios han descrito cambios en el metabolismo de calcio y magnesio en los casos de hipertensión arterial $\left({ }^{12-14}\right)$, también se determinó magnesio plasmático.

\section{MÉTODOS}

El estudio fue analítico observacional, tipo casos y controles.

Los pacientes y controles fueron seleccionados de una campaña de salud realizada en la sección Bioquímica y Biología Molecular, de la Facultad de Medicina, UNMSM. El grupo de estudio estuvo constituido por 40 personas, de ambos sexos, de edades entre 50 y 60 años, con una edad promedio de 57,3 años, residentes en la ciudad de Lima. Se trabajó con 20 pacientes hipertensos, medicados y controlados, con una presión arterial de $(143 \pm 3$ $/ 85 \pm 2 \mathrm{mmHg}$ ). Se excluyó del estudio a las personas con diabetes mellitus, artritis reumatoide, cardiopatías, nefropatías y dislipidemias u otras enfermedades crónicas. El grupo control estuvo constituido por 20 personas normotensas, con una presión arterial de $(120 \pm 0,3 / 78 \pm 0,2 \mathrm{mmHg})$ y con los mismos criterios de exclusión del grupo de hipertensos.

Después de 12 horas de ayuno, en ambos grupos se extrajo $4 \mathrm{~mL}$ de sangre del pliegue anterocubital. 
Se determinó los niveles de selenio en plasma, por el método espectrométrico de absorción atómica, el cual se basa en la reducción de selenito a seleniuro. Los niveles de zinc fueron determinados por espectrofotometría, midiendo la formación de un complejo coloreado a una absorbancia a longitud de onda de $560 \mathrm{~nm}$. Los niveles de magnesio fueron determinados utilizando el método colorimétrico, por formación de un complejo rojo-purpúrea, que se lee a 520nm. La formación del complejo es directamente proporcional al contenido de magnesio en la muestra.

La actividad de la enzima SOD en glóbulos rojos se evaluó de acuerdo a la técnica de Marklund y Marklund $\left({ }^{15}\right)$ y la peroxidación lipídica, según Suárez $\left({ }^{16}\right)$.

Todos los participantes en el estudio firmaron un consentimiento informado, aprobado por el Consejo Superior de Investigación de la UNMSM.

La diferencia de medias entre los grupos de estudio fue estimada mediante la prueba estadística t-student, a un valor de $p<0,05$.

\section{RESULTADOS}

En la Tabla 1, se observa que los niveles de selenio $(\mu \mathrm{mol} / \mathrm{L})$ no muestran diferencia significativa en ambos grupos de estudio. En cuanto a las concentraciones de magnesio (mg/dL) en ambos grupos, se halló diferencia significativa $(p<0,05)$, siendo los niveles elevados en el grupo de pacientes hipertensos.

Respecto a los valores de zinc $(\mu \mathrm{g} / \mathrm{dL})$, se observa una disminución significativa de las con-

Tabla 1. Niveles de zinc, magnesio y selenio en pacientes hipertensos y normotensos.

\begin{tabular}{lcc}
\hline & $\begin{array}{c}\text { Personas } \\
\text { normotensas }\end{array}$ & $\begin{array}{c}\text { Pacientes } \\
\text { hipertensos }\end{array}$ \\
\hline $\mathrm{Se}(\mu \mathrm{mol} / \mathrm{L})$ & $0,948 \pm 0,302$ & $0,884 \pm 0,105$ \\
$\mathrm{Mg}(\mathrm{mg} / \mathrm{dL})$ & $* 2,09 \pm 0,14$ & $* 2,85 \pm 0,36$ \\
$\mathrm{Zn}(\mu \mathrm{g} / \mathrm{dL})$ & $* 87,60 \pm 7,4$ & $* 75,88 \pm 9,3$ \\
\hline$n=20$ & \\
$*$ Existe diferencia de medias $p<0,05$ &
\end{tabular}

Tabla 2. Niveles de TBARS y superóxido dismutasa en pacientes hipertensos y normotensos.

\begin{tabular}{lcc}
\hline & $\begin{array}{c}\text { Personas } \\
\text { normotensas }\end{array}$ & $\begin{array}{c}\text { Pacientes } \\
\text { hipertensos }\end{array}$ \\
\hline MDA-TBARS $(\mu \mathrm{mol} / \mathrm{L})$ & $* 2,49 \pm 0,124$ & $* 2,83 \pm 0,36$ \\
$\mathrm{SOD}(\mathrm{U} / \mathrm{ml})$ & $* 918 \pm 84$ & $* 786 \pm 151$ \\
\hline $\mathrm{n}=20$ & \\
$*$ Existe diferencia de medias $p<0,05$ &
\end{tabular}

centraciones en el grupo de pacientes hipertensos, respecto al grupo control $(p<0,05)$.

Al evaluar las defensas antioxidantes en ambos grupos de estudio (Tabla 2), se encontró los niveles de la enzima SOD $(\mathrm{U} / \mathrm{ml})$ disminuidos con respecto al grupo control $(p<0,05)$.

Los valores del complejo coloreado MDATBARS $(\mu \mathrm{mol} / \mathrm{L})$ mostrados en la Tabla 2 revelan que el grupo de estudio, de pacientes hipertensos, muestra valores incrementados de este complejo, con una diferencia significativa $(p<0,05)$ con respecto al grupo control.

\section{DISCUSIÓN}

En estudios clínicos, se ha demostrado incremento en la producción de EROs en pacientes con hipertensión esencial. Sin embargo, cuando la hipertensión es leve o moderada y sin tratamiento, Ward $\left({ }^{10}\right)$ encontró que la peroxidación lipídica y el estrés oxidativo no están aumentados. Nuestros hallazgos muestran lo contrario. En el grupo de hipertensos leves $(143 \pm 3 / 85 \pm 2 \mathrm{mmHg})$ que recibían tratamiento, se observa un incremento de la peroxidación lipídica $(p<0,05)$ respecto al grupo control.

La determinación directa de EROs no es común en la clínica. La situación oxidativa del organismo es usualmente evaluada por el estado de las defensas antioxidantes (SOD, catalasa, glutatión reductasa, vitamina $\mathrm{E}$, selenio, zinc, manganeso y cobre) y por las consecuencias de las acciones de las EROs (lipoperoxidación, daño al ADN) $\left({ }^{17}\right)$.

En esta investigación, se encontró niveles disminuidos de zinc $(p<0,05)$ en pacientes hipertensos. 
Sin embargo, los niveles de selenio no presentaron diferencia significativa. También, se halló actividad significativamente disminuida de la SOD $(p<0,05)$, resultados que concuerdan con los estudios realizados por Redon y colaboradores $\left({ }^{5}\right)$. La SOD eritrocítica es una metaloenzima dependiente de zinc y cobre. Su actividad disminuida es desfavorable para los glóbulos rojos, puesto que este ambiente rico en oxígeno aumenta la posibilidad de formar el radical libre superóxido y disminuye la formación de peróxido de hidrógeno, molécula que podría ser eficientemente reducida por la glutatión peroxidasa o la catalasa. Esto podría explicar los valores similares de selenio, puesto que no se está demandando una mayor actividad de esta enzima.

El aumento del radical superóxido lleva a la formación del peroxinitrito, al reaccionar espontáneamente con el óxido nítrico (NO), anulando la función de este radical vasodilatador liberado por el endotelio. El peroxinitrito puede protonarse a ácido peroxinitroso, producto que está entre las especies más reactivas en los sistemas biológicos $\left({ }^{13}\right)$.

Chakraborty y colaboradores $\left({ }^{18}\right)$ han hallado una correlación inversa entre la actividad disminuida de la SOD y los niveles de TBARS. Este último parámetro, usado como marcador de peroxidación lipídica, también se ha hallado aumentado en el presente trabajo. Ciertamente, la pobre dismutación del superóxido provocará un incremento de reacciones con diversas estructuras, como por ejemplo los lípidos plasmáticos, y con las membranas de los elementos formes de la sangre. Este daño a membrana puede provocar la salida de elementos iónicos intracelulares, como el magnesio. Sin embargo, no podemos aseverar que esta injuria haya sido la causa de encontrar niveles significativamente elevados de magnesio en los pacientes hipertensos.

Diversos estudios $\left({ }^{19-21}\right)$ han mostrado que el éstres oxidativo constituye uno de los procesos involucrados en la HTA esencial, ya sea en la génesis o evolución de esta patología. En el presente estudio, los resultados también corroboran esta relación.

Los eventos bioquímicos relacionados con el daño oxidativo que se producen en la hipertensión, aún en casos leves, han mostrado el compromiso de injuria a nivel intraeritrocitario, que altera algunos parámetros plasmáticos, como el magnesio y el zinc. Será importante establecer una correlación entre otros oligoelementos séricos, que también participan en la fisiopatología de la hipertensión con enzimas intraeritrocitarias.

\section{REFERENCIAS BIBLIOGRÁFICAS}

1. Carranza J, Chávez F, Huape S, Moreno A, Camargo A, Hipertensión esencial enfermedad subdiagnosticada, pero ¿también sobreestimada? Rev Asoc Med Inte Mex. 1995:2(1):37-40.

2. Touyz R. Estrés oxidativo en la hipertensión arterial Boletín del Consejo Argentino de HTA. 2005;6:22-9.

3. Touyz R. Reactive oxygen species in vascular biology: role in arterial hypertension. Expert Rev Cardiovascular Ther. 2003;1:91-106.

4. Keshi T, Hirooka Y, Kimura Y, et al. Increased reactive oxygen species in rostral ventrolateral medulle contribute to neural mechanisms of hypertension in stroke-prone spontaneously hypertensive rats. Circulation. 2004;109:235762.

5. Redon J, Olivia M, Tormos C, Giner V, Chaves J, Iradi A, et al. Antioxidant activities and oxidative stress by products in human hypertension. Hypertension. 2003;41(5):1096.

6. Rodríguez-Iturbe B, Zhan CD, Quiroz Y, et al. Antioxidantrich diet relieves hypertension and reduces renal inmune infiltration in spontaneously hypertensive rats. Hypertension. 2003;41:341-6.

7. Vaziri ND, Wang XQ, Oveisi F, Rad B. Induction of oxidative stress by glutathione depletion causes severe hypertension in normal rats. Hypertension. 2000;36(1):142-6.

8. Dajas F, Ferrari A, Martinez A, Zeppi M, Ferreiras M, Pintos A. Producción de radicales hidroxilo en sangre en pacientes ancianos hipertensos. Rev Med Urug. 2004;20(1):12-8.

9. Russo C, Olivieri O, Girelli D, Faccini G, Zenari Ml, Lombardi S. Antioxidant status and lipid peroxidation in patiens with hyperetension. J Hypertens. 1998;16(9):1267-71.

10. Ward NC, Hodgson JM, Puddey IB, Mori TA, Beilin LJ, Croft KD. Oxidative stress in human hypertension: association with antihypertensive treatment, gender nutrition, and lifestyle. Free Radic Biol Med. 2004;36:226-32.

11. Sontia B, Touyz R. Role of magnesium in hypertension. Archives of Biochemistry and Biophysics. 2007;458(1):339.

12. Villanueva LA, Figueroa A, Villanueva S. Concentraciones séricas de electrolitos en mujeres con preeclampsia severa. Rev Hosp Gral Dr. M Gea González. 2000;3(4):166-9.

13. Kosch M, Hausberg M, Westermann G, Köneke J, Matzkies F, Heinz-Rahn K, et al. Alteraciones del contenido de calcio y magnesio de las membranas de los hematíes en pacientes con hipertensión primaria. Am J Hypertens. 2001;14:254-8. 
14. Laurant P, Touyz R, Physiological and pathophysiological role of magnesium in the cardiovascular system: implications in hipertension. J Hypertension. 2000;18(9):1177-91.

15. Marklund S, Markalund G. Involvement of the superoxide anion radical in the antioxidation of pyragallol and a convenient assay for superoxide dismutase. Eur J Biochem. 1974; 47:469-74.

16. Suárez CS. Detoxificación hepática y defensa antioxidante por efecto de xenobióticos alimentarios. Tesis de Maestría en Bioquímica. Facultad de Medicina, Universidad Nacional Mayor de San Marcos. Lima, Perú. 1995.

17. Cruz M, Sanfiel V, Maceo P. Estrés oxidativo e hipertensión esencial: una realidad clínica. Revista Cubana de Invest Biomed. 2004;23:3-9.

18. Chakraborty I, Kunti S, Bandyopadhyay M, Dasgupta A. Evaluation of serum zinc level and plasma SOD activity in senile cataract patients under oxidative stress. Indian Journal of Clinical Biochemistry. 2007;22(2):109-13.

19. Touyz RM. Reactive oxygen species, vascular oxidative stress, and redox signaling in hypertension: what is the clinical significance? Hypertension. 2004;44:248-52.
20. Pollock DM. Endothelin, angiotensin, and oxidative stress in hypertension. Hypertension. 2005;45:477.

21. $\mathrm{Pu} \mathrm{Q}$, Neves MF, Virdis A, Touyz RM, Schiffrin EL. Endothelin antagonism on aldosterone-induced oxidative stress and vascular remodeling. Hypertension. 2003;42:4955 .

Manuscrito recibido el 17 de octubre de 2007 y aceptado para publicación el 13 de diciembre de 2007.

Correspondencia:

$\mathrm{Mg}$. Raquel Oré Sifuentes

Centro de Investigación de Bioquímica y Nutrición Facultad de Medicina, UNMSM

Av. Grau 755. Lima 1, Perú

Correo-e: kelaore@yahoo.es 